\title{
Unrecognized Hypoglycemia Due to Maltodextrin Interference with Bedside Glucometry
}

\author{
Barbara M. Kirrane, MD ${ }^{a}$, Elizabeth A. Duthie, RN, PhD ${ }^{b}$, Lewis S. Nelson, $\mathrm{MD}^{c}$
}

aDepartment of Surgery, Section of Emergency Medicine. Yale University School of Medicine, New Haven, CT

bDirector of Patient Safety, NYU Langone Medical Center, New York, NY

cDepartment of Emergency Medicine, Medical Toxicology Fellowship, New York University School of Medicine, New York, NY

\begin{abstract}
Introduction: Glucometry is widely used to confirm or exclude hypoglycemia in patients with suggestive clinical findings. Nonglucose sugars may be detected by certain types of glucometers, causing false elevation of the glucometer analysis of the blood sugar. Since these other sugars are not functionally glucose and may even induce excess insulin release, clinical hypoglycemia may be missed.

Case Report: We report a 79-year-old man on enteral feeds containing maltodextrin, a glucose polymer, who had persistently high glucometer-measured blood glucose despite normal blood glucose measured by formal laboratory analysis.

Discussion: Excess insulin administration, based on the erroneous glucometer reading, may have caused unrecognized fatal clinical hypoglycemia. This has been reported following intravenous administration of related nonglucose sugars but not with enteral maltodextrin. Further study is required to confirm the effects of maltodextrin on glucometry.

Conclusion: False elevation of blood glucose measured on certain point-of-care glucometers can occur following the oral administration of maltodextrin.
\end{abstract}

\section{INTRODUCTION}

Portable point-of-care glucometer devices are often utilized to measure blood glucose in hospital settings. Portable bedside glucometers utilize enzymatic assays to provide a rapid measurement of capillary blood glucose, allowing for timely clinical decisions. While the accuracy of bedside glucometers has been extensively evaluated, false elevation of glucose measurements may occur when nonglucose sugars are present in the blood, such as galactose and maltose. Sugars may be exogenously introduced into the bloodstream for a variety of reasons, e.g., the absorption of icodextrin, a glucose polymer, which occurs while it is used in the dialysate solution for peritoneal dialysis. Accordingly, the US Food and Drug Administration issue a safety warning advising caution when using certain portable point-of-care monitors with patients receiving parenteral products containing maltose or galactose, and for oral xylose [1]. While false elevation of glucometry measurements have been reported in the aforementioned circumstances, it is not known to occur following the oral administration of maltose or its polymer, maltodextrin. We report a case of unrecognized fatal hypoglycemia in the setting of falsely elevated glucometer glucose concentration likely due to maltodextrin contained in enteral nutrition.

\section{CASE REPORT}

A 79 year-old-man with multiple medical problems, including insulin-dependent diabetes mellitus, was admitted to the hospital

Keywords: maltodextrin, hypoglycemia, point-of-care glucometers

Notes: This paper was presented as a poster at the September 2007 meeting of the North American Congress of Clinical Toxicology in San Francisco, CA.

There was no outside funding of any kind used for this study.

Corresponding Author: Barbara M. Kirrane, Department of Surgery, Section of Emergency Medicine, Yale University, 464 Congress Avenue, Suite 260, New Haven, CT 06511. E-mail: bmkirrane@gmail.com 


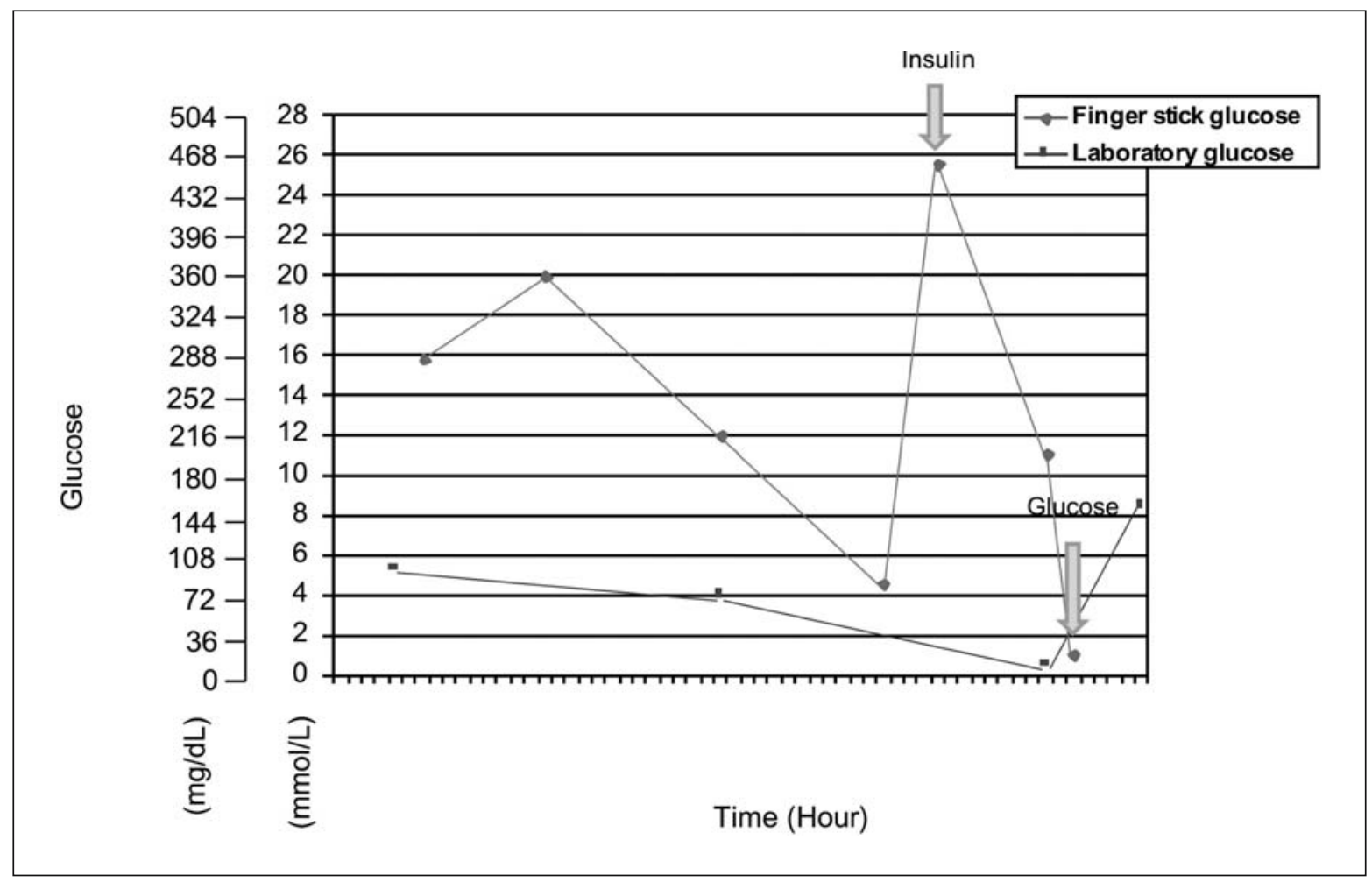

Figure 1: Fingerstick and serum glucose measurements over time.

for presumed sepsis. His medications prior to admission included furosemide $20 \mathrm{mg}$ once a day, losartan $50 \mathrm{mg}$ once a day, escitalopram $10 \mathrm{mg}$ once every day, omeprazole $20 \mathrm{mg}$ every day, lactobacillus, and potassium chloride $20 \mathrm{mEq}$ once a day. In addition to these medications, the patient also received vancomycin, cefepime, amikacin, voriconazole, and both regular insulin and glargine insulin during his inpatient stay. On hospital day 3 he was started on enteral feeds containing maltodextrin (Peptamen, Nestle Nutrition, Vevey, Switzerland) at $50 \mathrm{ml} / \mathrm{hr}$ (1200 ml/day) by nasogastric tube. A routine evening (21:41) glucometer glucose concentration was $25.6 \mathrm{mmol} / \mathrm{L}$ (461 mg/dL) as measured by the Roche Accu-chek Advantage portable glucometer, which utilizes the glucose dehydrogenase enzyme with pyrroloquinolinequinone (GDH-PQQ). Subsequently, sliding scale insulin was administered ( 24 units insulin glargine subcutaneous and 10 units regular insulin subcutaneous) for this elevated reading. The next morning (05:51) a glucometer measurement was $11.1 \mathrm{mmol} / \mathrm{L}(200 \mathrm{mg} / \mathrm{dL})$, and routine morning serum labs were sent simultaneously. The nursing notes indicated that the patient slept through the finger stick glucose check and blood draw. Approximately 1 hour later, the chemistry lab reported serum glucose of less than $1.11 \mathrm{mmol} / \mathrm{L}(<20$ $\mathrm{mg} / \mathrm{dL}$ ). Intravenous glucose did not provide improvement, and the patient subsequently expired. Review of the patient's glucometer glucose concentrations compared to laboratory glucose concentrations revealed a persistently elevated reading with discrepancy between the serum and bedside values (Figure 1).

\section{DISCUSSION}

Point-of-care glucometers use enzymatic assays to measure glucose concentrations. Several different enzymes are utilized by the various commercial glucometers, including glucose dehydrogenase, glucose oxidase, and hexose kinase. Analyzers using the glucose dehydrogenase system can be further subdivided based on cofactor utilized to catalyze the reaction: either nicotinamide adenine dinucleotide $\left(\mathrm{GDH}-\mathrm{NAD}^{+}\right)$or pyrroloquinolinequinone (GDH-PQQ). Interference with glucose measurement has been reported to occur most commonly on analyzers using the GDHPQQ enzyme [2].

Neonates with galactosemia who consequently had elevated serum galactose levels were found to have falsely elevated glucose concentrations detected on point-of-care analyzers that used glucose dehydrogenase enzymes [3]. In a subsequent study on whole blood, the error did not occur with analyzers that measured glucose by using hexose kinase enzymes or those that used glucose oxidase enzymes [2]. Diabetic patients using icodextrin containing peritoneal dialysis fluid have had falsely elevated glucose readings [4-6] Icodextrin, a polymer of glucose, is used in peritoneal dialysis as an osmotic agent to facilitate fluid movement across membranes. When used for this purpose, icodextrin may be absorbed by the lymphatic system, and then subsequently hydrolyzed to glucose oligomers such as maltose, maltriose and maltotetraose [4]. When in vitro blood samples spiked with icodextrin metabolites are analyzed on point-of-care glucometers, 


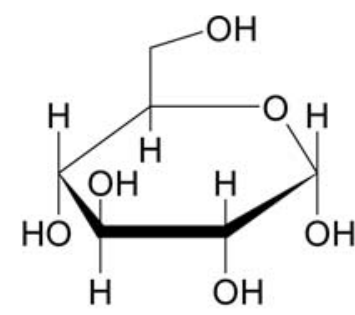

Glucose

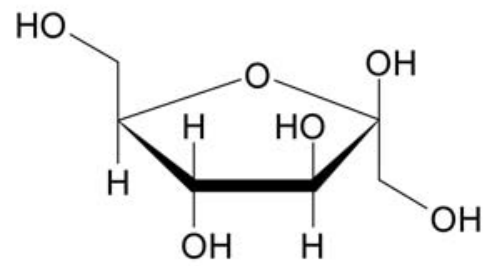

Fructose

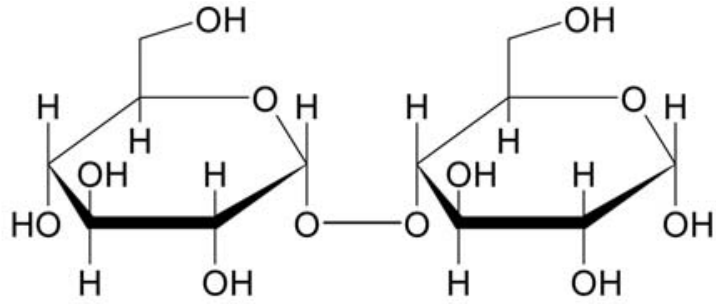

Maltose (Maltodextrin)

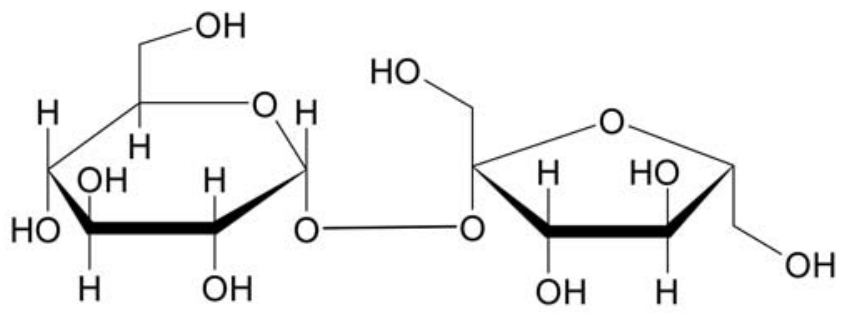

Sucrose

Figure 2: Chemical structures of similar sugars.

the false glucose elevation is reproduced $[2,4,7,8]$. This most consistently occurs on the GDH-PQQ analyzers, though in one study, interference was also found in a glucose oxidase glucometer [7]. Similar situations of falsely elevated glucose have occurred in patients receiving other maltose containing therapy. Recently, a patient receiving intravenous human immunoglobin (Octagam, Octapharma AG, Lachen, Switzerland), which contains maltose, had falsely elevated glucose values measured by a portable glucometer (glucose dehydrogenase), consequently was placed on high-dose insulin, and developed profound hypoglycemia [9]. Though the exact mechanism of interference is not yet specifically defined, it is likely caused by lack of enzyme specificity, due to the similarity in structure between glucose and other sugars, such as maltose and galactose (Figure 2).

Enteral feeds, such as Peptamen, introduce oligosaccharides, such as maltodextrin, into the gastrointestinal (GI) tract. Maltodextrin is a sweet polysaccharide that is used to provide both thickening to the feed texture and caloric content. Digestion is initiated rapidly in the GI tract by carbohydrate-specific hydrolyases bound to the brush border of the small intestine. Maltodextrin is generally not absorbed whole, but instead is digested to monosaccharide components prior to transport across the surface membrane of the intestinal epithelial cells.

Under normal circumstances, disaccharides do not passively cross the intestinal barrier. Sucrose, a disaccharide of glucose and fructose, is not absorbed through the mucosa of an intact GI tract, but may be absorbed in the presence of barrier breakdown, such as ulcers, inflammation and atrophic gastritis [10,11]. It is presumed that as with sucrose, maltodextrin-or a smaller oligosaccharide metabolite or maltose-may be absorbed by passive diffusion in the setting of gastric inflammation.
Interestingly, nonglucose sugars may stimulate endogenous insulin release. When given by intravenous infusion to dogs, xylitol, a 5-carbon sugar alcohol, resulted in measurable increases in insulin secretion [12]. This presumably occurs as a result of structural similarity between xylitol and glucose. Although endogenous insulin levels were not measured on our patient, the hypoglycemia in this case was likely to have occurred from the administration of exogenous insulin, administered on the basis of an elevated bedside glucometer reading.

Additionally, it is possible that the feeding solutions were a contaminant on the patientís hand and resulted in external interference rather than reflecting absorption. However, this is unlikely, as skin is routinely cleaned with alcohol swabs prior to glucose testing and due to the consistent finding on repetitive analyses.

\section{CONCLUSION}

As with other nonglucose sugars, false elevation of blood glucose measured on certain point-of-care glucometers can occur following the oral administration of maltodextrin. Further studies should evaluate the likelihood of this problem based on the specific glucometer type used for the measurement.

The authors have no potential financial conflicts of interest to report.

\section{REFERENCES}

1. US Food and Drug Administration, [web page on the Internet]. Important safety information on interference with blood glucose measurement following use of parenteral 
maltose/parenteral galactose/oral-xylose-containing products [cited 2007 November 19]. Available at: http://www.fda.gov/ cber/safety/maltose110405.htm

2. Ghys T, Goedhuys W, Spincemaille K, Gorus F, Gerlo E. Plasma-equivalent glucose at the point-of-care: evaluation of Roche Accu-Chek Inform and Abbott Precision PCx glucose meters. Clin Chim Acta 2007;386:63-68.

3. Newman JD, Ramsden CA, Balazs NDH. Monitoring neonatal hypoglycemia with the Accu-chek Advantage II glucose meter: The cautionary tale of galactosemia. Clin Chem 2002;48:2071.

4. Apperloo JJ, Vader HL. A quantitative appraisal of interference by icodextrin metabolites in point-of-care glucose analyses. Clin Chem Lab Med 2005;43:314-318.

5. Mehmet S, Quan G, Thomas S, et al. Important causes of hypoglycemia in patients with diabetes on peritoneal dialysis. Diabet Med 2001;18:679-682.

6. Riley SG, Chess J, Donovan KL, et al. Spurious hyperglycaemia and icodextrin in peritoneal dialysis fluid. BMJ 2003; 327:608-609.
7. Janssen W, Harff G, Caers M, et al. Positive interferences of icodextrin metabolites in some enzymatic glucose methods. Clin Chem 1998;44:2379-2380.

8. Wang R, Skoufos L, Martis L. Glucose monitoring for diabetic patients using icodextrin. Perit Dial Int 2004;24: 296-297.

9. ISMP Medication Safety Alert. Be aware of false glucose results with point-of-care testing. 2005;10:1-3.

10. Sutherland LR, Verhoef M, Wallace JL, et al. A simple, non-invasive marker of gastric damage: sucrose permeability. Lancet 1994;343:998-1000.

11. Sjöstedt Zsigmond C, Hannestad U, Franzén L, et al. Atrophic gastritis is associated with increased sucrose permeability related to chronic inflammation. Digestion 2005; 72:201-206.

12. Asano T, Greenberg BZ, Wittmers PV, et al. Xylitol, a partial homologue of alpha-D-glucopyranose: a potent stimulator of insulin release in dogs. Endocrinology 1977;100: 339-345. 